Ann. Zootech., I964, 13 (4), 34I-35o.

\title{
INFLUENCE DE L'ACRYLATE DE SODIUM SUR LA CROISSANCE DU PORCELET
}

\author{
M. MICHEL, C. JOUANDET, E. SALMON-LEGAGNEUR, A. AUMAITRE \\ et A. C. FRANÇOIS \\ Avec la collaboration technique de M. Guichard, Simone Boche \\ et Genevieve HaxNeqQUarT \\ Station centrale de Nutrition, \\ Station de Recherches sur l'lilevage des Porcs, \\ Centre national de Recherches zootechniques, Jouy-en-Josas (Seine-et-Oise)
}

\section{SOMMAIRE:}

L'influence de l'acrylate de sodium sur la croissance du porcelet est étudiée sur I I portées de o à 9 semaines.

Pendant la période d'allaitement (de o à 5 semaines), l'acrylate ne provoque aucune amélio ration; par contre, après le sevrage, (de 5 à 9 semaines), on note une augmentation de 16 p. Ioo du gain de poids et de I 3 p. soo de l'efficacité alimentaire et une diminution sensible de la fréquence des diarrhées. Ces résultats sont comparables ì ceux obscrvés avec l'oxytétracycline dans les mêmes conditions.

Le dosage dans les fèces de quelques substances azotées basiques (lysine, cadavérine, putrescine, tyramine et ammoniac) permet de préciser un aspect des relations entre l'inhibition du catabolisme azoté de la flore intestinale et la stimulation de croissance obtenue avec l'acrylate de sodium ou l'oxytétracycilne.

\section{IN'TRODUCTION}

SIFBUR'TH (I960) a souligné le premier la relation qui semblait exister entre l'absence de certains germes microbiens dans le tube digestif du pingouin et l'ingesgestion de plancton riche en acide acrylique.

Depuis cette observation, quelques expériences ont été entreprises sur les applications nutritionnelles que pouvaient présenter les sels de cet acide chez différentes espèces.

Ainsi WhITE-STEVENs et al. (I962) ont montré que les sels de sodium, de potassium et de magnésium de l'acide acrylique amélioraient la vitesse de croissance 
du poussin, tandis que les sels de zinc ou de calcium étaient sans action. De même, WALlace et Combs (I962) ont administré de l'acrylate de sodium à des porcs. en croissance avec des résultats divers.

Le mécanisme que l'on pourrait invoquer pour expliquer l'influence de l'acideacrylique, ou de ses sels, sur la croissance est analogue à celui que l'on retient parfois pour les antibiotiques : diminution de l'activité catabolique d'une partie de la flore intestinale et notamment inhibition de la dégradation des acides aminés. En effet, l'ingestion de tétracycline par le porc abaisse significativement le taux d'ammoniac du sang de la veine porte (Françors et Michel, I960), ainsi que la. formation d'amines au niveau de l'iléon (LARSOx et HII,L, I960). L'acide acrylique, par son action inhibitrice de la croissance des coliformes au niveau intestinal (SreBurTh, I96I), pourrait exercer un effet analogue.

Il était alors intéressant d'examiner quelle serait l'action de telles substances chez le très jeune porcelet, car celui-ci présente au moment du serrage de profonds. bouleversements de son faciès bactérien intestinal qui s'accompagnent d'une sensibilité accrue à tous les facteurs extérieurs. (Wri,Bur et al. x960).

A cet effet, nous avons entrepris une expérience pour comparer sur des porcelets allaités, puis en cours de sevrage, l'influence d'une distribution orale d'acrylate de sodium ou d'oxytétracycline, antibiotique comnu pour la stimulation de croissance qu'il provoque. Dans cette étude, nous avons pris en considération non seulement les différences de vitesse de croissance des animaux et d'utilisation des aliments, mais encore les modifications des propriétés biochimiques de la flore intestinale qui pouvaient se rattacher à cette action.

I)ans cette optique, nous avons cherché à déterminer si l'excrétion de certaines formes d'azote pouvait représenter un témoin valable de l'activité métabolique de la flore intestinale et, d'autre part, à établir une relation entre cette activité et la vitesse de croissance de l'animal.

\section{MÉTHODE}

\section{A. - Priparation de l'acrylate de sodium}

L'acide acrylique glacial, contenant 2 p. roo d'hydroquinone, est d'abord additioné de carbonate de sodium anhydre $(200 \mathrm{~g} / \mathrm{kg})$ puis de soude à $40 \mathrm{p}$. Ioo pour atteindre la neutralité. Toutes ces opérations sont effectuées sous agitation et en refroidissant par de l'eau glacée. Ce procédé ne convient que pour de faibles quantités, inférieures à $\mathrm{I} \mathrm{kg}$ : dans une préparation une faible polymérisation s'est produite avec formation de substances gélatineuses.

\section{B. - Animaux}

Onze portées de porcelets de race Large White sont réparties dès la naissance en 3 lots semblables quant au poids et au sexe. L'un des lots sert de témoin, tandis que les deux autres reçoivent soit de l'acrylate de sodium, soit de l'oxytétracycline, de la façon suivante :

$\mathrm{I}^{0}$ de la naissance à 5 semaines, période d'allaitement, la distribution se fait par ingestion forcée d'une solution contenant suivant les lots :

- r6o mg de glucose par ml (lot témoin),

- I g d'acrylate de sodium par ml (lot acrylate),

- $20 \mathrm{mg}$ de chlorhydrate d'oxytétracycline sur un support de $\mathrm{r} 60 \mathrm{mg}$ de glucose par ml (lot oxytétracycline).

Ces solutions sont administrées trois fois par semaine à raison de $\mathrm{I} \mathrm{ml}$ pour $2 \mathrm{~kg}$ de poids vif. 
Pendant cette période, les porcelets ont à leur disposition de l'eau et un aliment pré-sevrage de type classique (1).

$2^{0}$ de 5 à 9 semaines, période du sevrage proprement dit, les animaux sont séparés de leur mère et consomment à volonté le même aliment que précédemment, auquel on incorpore directement suivant les lots :

- $2 \mathrm{~g}$ de glucose par $\mathrm{kg}$ d'aliment :-

- $2 \mathrm{~g}$ d'acrylate de sodium par $\mathrm{kg}$ d'aliment ;

- $40 \mathrm{mg}$ de chlorhydrate d'oxytétracycline et $2 \mathrm{~g}$ de glucose par $\mathrm{kg}$ d'aliment.

Les poids des animaux aux différents stades, les quantités d'aliments consommées par semaine sont notées pour chaque lot. Des observations sont faites sur l'état sanitaire apparent des porcelets (nombre de jours de diarrhées par porcelet). La signification des différences observées dans les résultats est testée par analyse de variance.

Par ailleurs, des échantillons hebdomadaires de fèces sont prélevés pour chaque lot de chaque portée. Les échantillons conservés à - I $5^{\circ} \mathrm{C}$ jusqu'à la fin de l'expérience permettent la constitution d'échantillons moyens par semaine et par lot. Ces derniers donnent lieu à la détermination des concentrations en diverses substances azotées basiques.

Enfin, le dosage de l'activité antibiotique des fèces du lot oxytétracycline a été effectué ; cela nous a permis de contrôler l'efficacité du système de distribution.

\section{C. - Dosage des substances azotées basiques des fèces (acides aminés basiques et amines)}

Il est effectué par séparation sur résine échangeuse de cations et comprend les opérations suivantes :

a) Mise en solution de l'échantillon à doser.

A $50 \mathrm{~g}$ d'un échantillon moyen de fèces pesé exactement, on ajoute $200 \mathrm{ml}$ d'alcool à $95^{\circ}$; après homogénéisation, on laisse une nuit à - I $5^{\circ} \mathrm{C}$. La suspension est filtrée avec l'aide de supercel et le filtre rincé par $5^{\circ} \mathrm{ml}$ d'alcool. Le filtrat est séché sous un courant d'air, à $30^{\circ} \mathrm{C}$. On reprend le résidu par $50 \mathrm{ml}$ de tampon citrate $0,2 \mathrm{~N}$. L'insoluble est séparé sur verre fritté. Le filtrat est stocké à - $5^{\circ} \mathrm{C}$ jusqu'au dosage.

\section{b) Chromatographie.}

On utilise une colonne $(0,8 \times 60 \mathrm{~cm})$ de résine Bio-Rex $70(<400$ mesh) importée par les Éts Eurobio, Paris.

On place avec précaution sur la résine $\mathrm{I} \mathrm{ml}$ de la solution à doser (correspondant à $\mathrm{I} g$ de fèces frais). Après passage, on rince avec du tampon $0,2 \mathrm{~N}$ (ro $\mathrm{ml}$ ) et on développe ensuite par un gradient du même tampon de 0,2 à $2 \mathrm{~N}$.

La vitesse de passage est de $10 \mathrm{ml}$ à l'heure. L'effluent recueilli par fraction de I $\mathrm{ml}$ est analysé selon Moore et STEIN (1954).

Tous les résultats sont exprimés en micromolécules par gramme de poids sec.

\section{RÉSULTATS}

\section{I. - Croissance et état sanitaire.}

Le tableau I donne les principaux résultats de l'expérience et les figures I et 2 permettent de suivre la croissance et l'évolution de l'état sanitaire (fréquence des diarrhées) at1 cours de 1'expérience.

On remarque en particulier que pendant la période d'alimentation essentiellement lactée, l'acrylate de sodium ne semble pas exercer d'action sur la vitesse de croissance. Il se différencie en cela de l'oxytétracycline qui provoque une légère

(1) Composition de l'aliment : Avoine décortiquée : 20 ; Blé : 20 ; Sucre : ro ; Manioc : ıo ; Saindoux : 7 ; Tourteau de soja : 7 ; Farine de poisson : 8 ; Levure : 5 ; Lait écrémé : to ; Composé minéral vitaminé : 3 . 
344 M. MICHEI, C. JOUANDET, F. SALMON-LEGAGNEUR, A. AUMAITRE, A. C. FRANÇOIS. augmentation du gain journalier. Celle-ci apparaît nettement sur les courbes de croissance à partir de la troisième semaine d'allaitement.

TABLEAU I

Croissance et état sanitaire des animaux

\begin{tabular}{|c|c|c|c|c|c|}
\hline \multirow[b]{2}{*}{ Lot } & \multirow[b]{2}{*}{ Témoin } & \multirow[b]{2}{*}{$\begin{array}{l}\text { Acrylate de } \\
\text { sodium }\end{array}$} & \multirow[b]{2}{*}{$\begin{array}{l}\text { Oxytétra- } \\
\text { cycline }\end{array}$} & \multicolumn{2}{|c|}{ Signification des différences } \\
\hline & & & & \begin{tabular}{|} 
Entre le lot \\
témoin et les lots \\
expérimentaux
\end{tabular} & $\begin{array}{l}\text { Entre le lot acry } \\
\text { late et le lot } \\
\text { oxytétracycline }\end{array}$ \\
\hline Nombre de porcelets .... & 31 & 31 & 31 & & \\
\hline Poids moyen à la naissance $(\mathrm{kg})$ & 1,3 & 1,2 & 1,2 & NS & NS \\
\hline Poids moyen à 5 semaines $(\mathrm{kg})$.. & 7,3 & 7,0 & 7,7 & NS & 0,05 \\
\hline Poids moyen à 9 semaines $(\mathrm{kg})$. . & 17,9 & 19,5 & 20 & 0,01 & NS \\
\hline $\begin{array}{l}\text { Gain de poids moyen quotidien de } \\
0 \text { à } 5 \text { semaines }(g) \ldots \ldots \ldots \ldots\end{array}$ & 171 & 165 & 185 & NS & 0,05 \\
\hline $\begin{array}{r}\text { Gain de poids moyen quotidien } \\
\text { de } 5 \text { à } 9 \text { semaines }(\mathrm{g}) \ldots \ldots \ldots\end{array}$ & 381 & 445 & 441 & 0,01 & NS \\
\hline $\begin{array}{r}\text { Consommation moyenne d'ali- } \\
\text { ment par porcelet de } 5 \text { à } 9 \text { sem- } \\
\text { maines }(\mathrm{kg}) \ldots \ldots \ldots \ldots \ldots \ldots \ldots\end{array}$ & 18,4 & 19,4 & 19,5 & NS & NS \\
\hline $\begin{array}{r}\text { Efficacité alimentaire (kg de gain } \\
\text { par kg d'aliment) } \ldots \ldots \ldots \ldots \ldots\end{array}$ & 0,565 & 0,632 & 0,641 & 0,01 & NS \\
\hline $\begin{array}{l}\text { Jours de diarrhée par porcelet de } \\
0 \text { à } 5 \text { semaines } \ldots \ldots \ldots \ldots \ldots\end{array}$ & 1,8 & 1,3 & 1,6 & NS & NS \\
\hline $\begin{array}{l}\text { Jours de diarrhée par porcelet de } \\
5 \text { à } 9 \text { semaines } \ldots \ldots \ldots \ldots \ldots\end{array}$ & & C & 54 & NS & NS \\
\hline Mortalité.................... & $\begin{array}{c}2 \text { par } \\
\text { écrasement }\end{array}$ & 0 & 0 & NS & NS \\
\hline
\end{tabular}

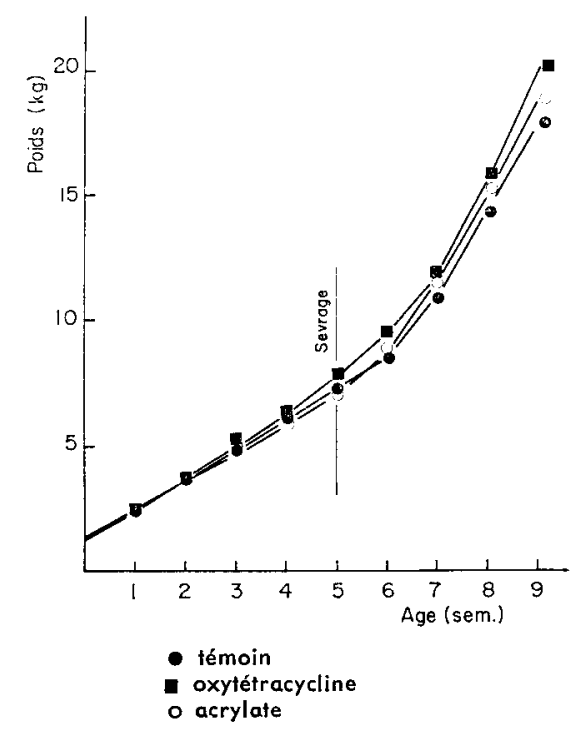

FIG. I. 
Par contre, de 5 à 9 semaines, c'est-à-dire après le sevrage, la stimulation de croissance est importante et du même ordre dans les deux lots expérimentaux. C'est ainsi que l'on observe une augmentation de $15 \mathrm{p}$. Ioo du gain de poids, par rapport au témoin, chez les animaux traités à l'oxytétracycline et de 16 p. Ioo chez les animaux traités à l'acrylate. En dépit d'une légère augmentation de la consommation d'aliment, on remarque que dans les deux lots les efficacités alimentaires sont respectivement supérieures à celles du lot témoin de I2 et I3 p. Ioo.

En ce qui concerne l'état sanitaire (fig. 2), on ne note pas de différence sensible de o à 5 semaines. Au sevrage, on observe une augmentation importante de la fréquence des diarrhées dans tous les lots, mais celle-ci est moins élevée dans les lots acrylate et oxytétracycline que dans le lot témoin. Il est d'ailleurs à remarquer que
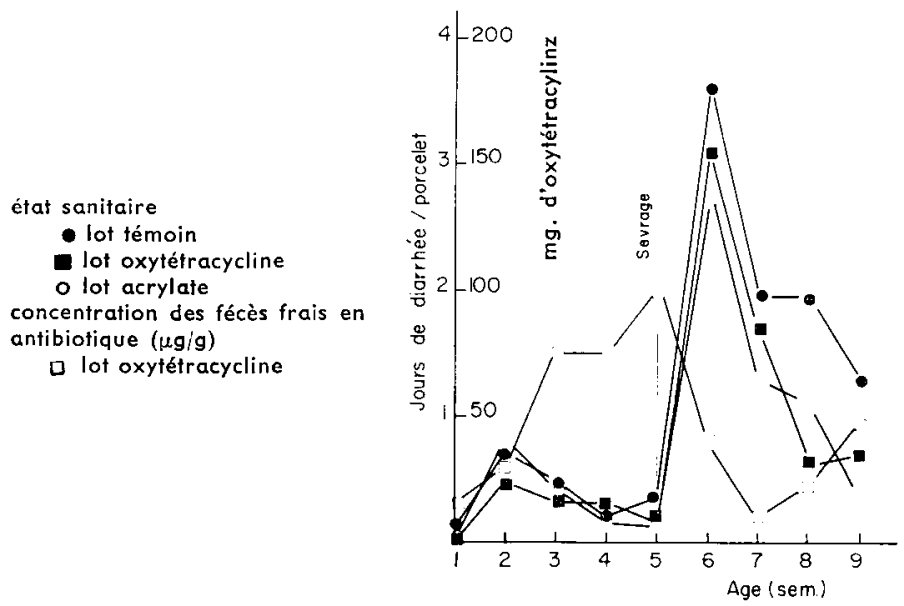

FIG. 2.

pour le lot oxytétracycline, la fréquence des diarrhées reflète assez bien la variation de la concentration en antibiotique des fèces. Par la suite, la fréquence des diarrhées diminue dans les 3 lots, mais plus rapidement dans les lots expérimenta11x.

\section{2. - Aspect biochimique de l'excrétion azotée fécale.}

\section{A) Animaux normanx.}

La nature et la concentration des substances azotées des fèces varient notablement suivant l'âge et l'état physiologique de l'animal. Avant le sevrage, la teneur en ammoniac est faible et celle en amines pratiquement nulle. Le changement de régime à 5 semaines est accompagné d'une augmentation considérable de l'excrétion des substances azotées basiques, ce qui indique une perturbation dans les phénomènes de la digestion.

Le tableau 2 résume les principaux résultats pour les 3 lots et la figure 3 permet de suivre l'évolution des différentes fractions isolées.

a) Acides aminés basiques.

La lysine est le seul acide aminé basique retrouvé dans les fèces: 1 'histidine et l'arginine font défaut ou existent à l'état de traces. En revanche, l'ornithine, proba- 
blement produite par la déguanidylation de l'arginine par la flore, existe dans presque tous les échantillons. L'excrétion de la lysine subit une augmentation notable au moment du sevrage, pour revenir ensuite à son niveau primitif. L'acrylate et l'oxytétracycline limitent l'ampleur de ces modifications (fig. 3).

\section{TABLEAU 2}

Excrétion de certaines formes d'azote fécal (micromolécules de substance par $\mathrm{g}$ de poids sec valeurs moyennes de 5 à 9 semaines).

\begin{tabular}{|c|c|c|c|}
\hline Lot & Témoin & Acrylate & $\begin{array}{c}\text { Oxytétra- } \\
\text { cycline }\end{array}$ \\
\hline $\begin{array}{l}\text { Teneur en matière sèche } \\
(\mathrm{p} .100) \ldots \ldots \ldots \ldots\end{array}$ & 32,9 & 32,3 & 32,9 \\
\hline Lysine . & 1,0 & $0,8^{\prime} \pm$ & 0,9 \\
\hline Cadavérine ........... & 7,8 & 7,0 & 9,9 \\
\hline Putrescine .... & 0,5 & 0,9 & 1,0 \\
\hline Tyramine..... & 0,7 & 0,4 & 0,5 \\
\hline Ammoniac ..... & 43,1 & 17,9 & 37,1 \\
\hline
\end{tabular}

b) Cadavérine.

Cette diamine est produite par la décarboxylation de la lysine par diverses souches microbiennes présentes au niveau intestinal (entérobactéries et streptocoques en particulier). Sa concentration, presque nulle dans les fèces à $1 \mathrm{a} 5^{\mathrm{e}}$ semaine, croît rapidement ensuite. A la $9^{\mathrm{e}}$ semaine, elle est plus élevée dans le lot oxytétracycline. La quantité excrétée est de l'ordre de dix fois celle de la lysine (fig. 3).

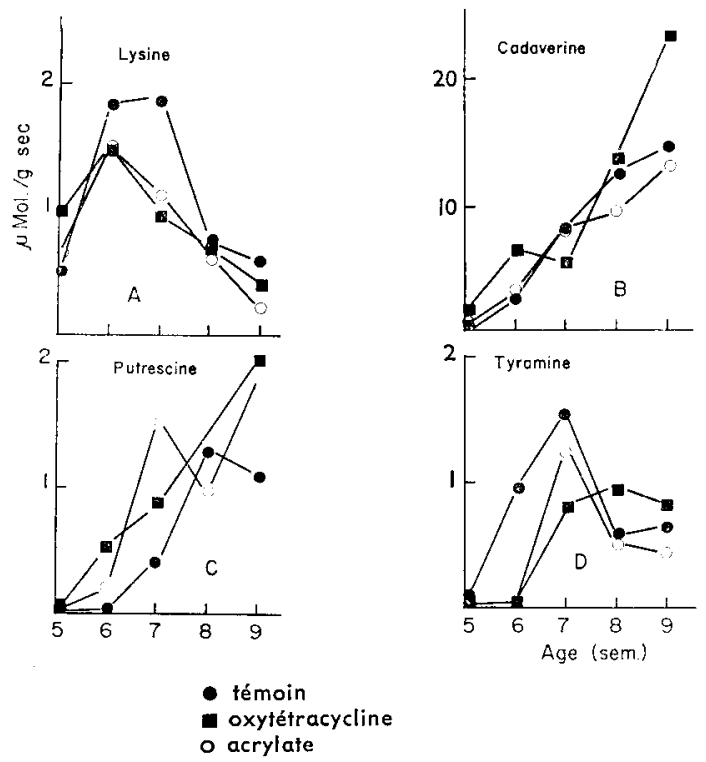

FIG. 3 . 
c) Putrescine.

Ce produit de la décarboxylation de l'ornithine, présent dans les fèces à une concentration de l'ordre de I/ro de celle de la cadavérine, suit la même progression que cette dernière. La teneur des lots "acrylate " et "oxytétracycline " est un peu plus élevée que celle des témoins (fig. 3).

d) Tyramine :

L'évolution de la concentration de cette substance est assez différente de celle des diamines. Chez les témoins, elle augmente dès la $5^{\mathrm{e}}$ semaine et décroît ensuite à partir de la $7^{\mathrm{e}}$ semaine. Chez les animaux traités, la teneur est plus faible et l'augmentation s'établit moins rapidement (fig. 3).

e) Ammoniac.

Les variations de la concentration en ammoniac, après le sevrage, sont moins accentuées. L'acrylate de sodium, plus que l'oxytétracycline, diminue fortement sa teneur dans les fèces.

\section{f) Autres substances.}

Ein dehors de 1'ornithine, déjà citée. d'autres bases azotées se trouvent dans les fèces : éthanolamine, agmatine, histamine, ainsi que trois substances non identifiées. Leur concentration est toujours très faible $(<0, \mathbf{I}$ micromolécule par $\mathrm{g}$ de poids sec.) Leur dosage étant peu précis dans ces conditions, 'les résultats n'ont pas été reportés.

\section{B) Infuence de l'état diarrhéique sur l'excrétion azotée fécale.}

Au cours des diarrhées, les fèces contiennent, par rapport au poids sec, des quantités beaucoup plus importantes de substances azotées que chez les animaux sains.

Le tableau 3 indique les valeurs obtenues pour la période de 7 à 9 semaines.

TABLEAU 3

Influence des diarrhées sur l'excrétion azotée fécale de 7 à 9 semaines

(valeurs moyennes en micromoles/g sec)

\begin{tabular}{|c|c|c|c|c|c|c|}
\hline \multirow{2}{*}{ Lot } & \multicolumn{3}{|c|}{ Animaux nomaux } & \multicolumn{3}{|c|}{ Diarrhées } \\
\hline & Témoin & Acrylate & Oxytétracycline & Témoin & Acrylate & Oxytétracycline \\
\hline Poids sec p. 100 & 28,8 & 28,6 & 29,4 & $11_{s} 1$ & 12,9 & 15,1 \\
\hline Lysine .......... & 1,06 & 0,69 & 0,73 & 3,75 & 3,03 & 3,66 \\
\hline Cadavérine ........ & 12,08 & 10,20 & 14,06 & 21,30 & 26,60 & 30,10 \\
\hline Tyramine... & 0,91 & 0,75 & 0,90 & 1,85 & 1,87 & 2,10 \\
\hline Ammoniac ......... & 56,7 & 16,2 & 33,0 & 199,0 & 179,0 & 223,0 \\
\hline
\end{tabular}

Annales de Zootechnie. - I964. 
Ces résultats mettent en évidence les faits suivants :

Io) l'absorption intestinale est nettement diminuée pendant la diarrhée, ainsi que l'indique l'augmentation d'environ 4 fois du taux de lysine ;

$\left.2^{\circ}\right)$ Le catabolisme azoté microbien est, accru surtout chez les animaux traités : la concentration en ammoniac est multipliée par II dans le lot acrylate contre 3,5 dans le lot témoin, mais elle demeure toujours plus faible dans le lot acrylate.

\section{DISCUSSION}

Le fait que le poids des porcelets à 9 semaines soit significativement supérieur dans les lots qui ont reçu de l'acrylate ou de l'oxytétracycline à celui des porcelets du lot témoin constitue une preuve de la stimulation de croissance provoquée par ces substances.

Celle-ci est toutefois sensiblement moins élevée que celle que nous avions rencontrée au cours d'expériences antérieures avec d'autres antibiotiques (SAI,MON-I,EGAGNEUR et Michel, I955; SALMON-LEGANEUR, I96r), mais dans des conditions différentes. Les conditions optimum d'emploi de l'acrylate restent d'ailleurs à préciser. Ėn outre, si l'influence de l'acrylate de sodium sur la vitesse de croissance du porcelet semble du même ordre que celle de l'oxytétracycline, ceci ne nous autorise pas à conclure que ce produit se comporte exactement comme un antibiotique.

En effet, l'amélioration due à l'acrylate apparaît seulement après le sevrage, alors que l'influence de l'oxytétracycline paraît plus précoce. Ceci peut être l'indice d'une différence de mode d'action, soit que les facteurs sur lesquels l'acrylate et l'antibiotique agissent ne soient pas les mêmes, soit que les conditions d'activité différent. Ceci n'est peut être pas sans rapport avec la nature et l'équilibre des régimes alimentaires qui varient profondément entre l'allaitement et le sevrage : en effet, jusqu'à 5 semaines, le régime alimentaire est presque exclusivement lacté et sans doute bien équilibré, alors qu'après le sevrage le régime est synthétique et probablement moins bien adapté.

Dans la mesure où les variations de l'excrétion de certaines substances azotées renseignent sur les différences du métabolisme et de l'efficacité alimentaire, on peut trouver un certain parallélisme entre les stimulations de croissance observées et l'action de l'acrylate, ou de l'antibiotique, sur la production d'ammoniac au niveau intestinal.

Nos résultats mettent en effet en évidence certaines perturbations biochimiques dans le tractus digestif, variables avec les traitements et particulièrement importantes au sevrage, c'est-à-dire au moment où les stimulations de croissance sont les plus élevées.

C'est ainsi que l'on voit apparaître à partir de la sixième semaine des quantités importantes de bases azotées provenant de la dégradation microbienne des acides aminés, mais celles-ci sont en général moins élevées notamment pour la tyramine et l'ammoniac dans les detux lots expérimentaux que dans le lot témoin. La limitation du taux d'ammoniac excrété est particulièrement nette dans le lot 
acrylate qui présente précisément la plus grande vitesse de croissance après le sevrage. Ce phénomène est à rapprocher de nos observations antérieures (MICHEL et FRANÇOIS, I955; SALMON-LEG.AGNEUR et Micheir, I955).

Qualitativement, nous avons retrouvé dans les fèces les mêmes amines que celles caractérisées par LARSON et HILL (I960) dans l'iléon du porcelet et cette répartition ne semble affectée ni par l'acrylate ni. par l'oxy tétracycline. Une différence avec Ies travaux de ces auteurs porte sur les concentrations respectives des diverses amines. Dans les fèces de tous nos animaux, la concentration en cadavérine est dix à vingt fois plus élevée que celle des autres bases, ce qui n'apparaît pas dans les résultats de LARSON au niveau de l'iléon. La cadavérine provenant de la lysine, il reste à préciser la signification nutritionnelle de la dégradation de cet acide aminé en fonction de l'âge, du régime et de la substance ajoutée. Une autre différence porte sur la formation des amines qui ne paraît affectée ni par l'acrylate, ni par l'oxytétracycline, du moins dans la partie postérieure du tractus intestinal. Dans cette partie, c'est surtout la formation d'ammoniac qui est diminuée.

Il faut noter que cette inhibition ne s'effectue plus pendant les diarrhées bien que la durée moyenne de celles-ci soit diminuée par l'oxytétracycline et l'acrylate.

En conclusion, ces observations préliminaires confirment nos résultats précédents, quant à la corrélation entre l'inhibition d'une partie du catabolisme azoté de la flore intestinale et la stimulation de croissance obtenue avec l'acrylate de sodium ou l'oxytétracycline, mais les différences de comportement des animaux soumis à ces deux traitements sont faibles et portent essentiellement sur la chronologie de la stimulation. Des mesures étendues à la totalité du tractus intestinal permettront de mieux préciser le mécanisme de cette action.

Reçu pour publication en juin 1964 .

\section{SUMMARY}

INFLUENCE OF SODIUM ACRYLATE ON THE GROWTH OF PIGLETS

The influence of sodium acrylate on the growth of piglets from birth to 9 weeks of age was studied in I I litters, each divided into three groups comparable in weight and sex. Group I was the control group, given glucose, group II received sodium acrylate and group III received oxytetracycline.

The treatments were :

from $\circ$ to 5 weeks, by forced feeding 3 times a week, respectively per $\mathrm{kg}$ live weight : $80 \mathrm{mg}$ glucose, $0.5 \mathrm{~g}$ acrylate or 10 $\mathrm{mg}$ oxytetracycline.

from 5 to 9 weeks, by direct incorporation into the feed, $2 \mathrm{~g}$ glucose, $2 \mathrm{~g}$ acrylate or $400 \mathrm{mg}$ oxytetracycline per $\mathrm{kg}$ feed.

During the suckling period sodium acrylate did not give any improvement in growth rate. In contrast, after weaning there was an increase of $16 \mathrm{p} .100$ in weight gain and of $13 \mathrm{p}$. 100 in efficiency of feed utilisation, and frequency of diarrhoea was markedly less compared with the control group. These results are comparable to those obtained with oxytetracycline.

Basic nitrogenous substances (lysine, cadaverine, putrescine, tyramine and ammoniac) were estimated in averaged weekly samples of faeces (table 2). It is possible from them to clarify one aspect of the relations between the inhibition of nitrogen catabolism by the intestinal microflora and the stimulation of growth obtained with sodium acrylate and oxytetracycline. These relations are discussed. 


\section{REMERCIEMENTS}

Nous adressons nos sincères remerciements à la Société industrielle de Biochimie et à la Société de Chimie organique et biologique pour leur contribution à la réalisation matérielle de cette expérience.

\section{RÉFÉRENCES BIBLIOGRAPHIQUES}

François A. C., Mrchel M., ig60. Effets métaboliques de certaines flores digestives. Conséquences nutritionnelles. Cah. Coll. Hôp. Exp. Scient. éd. Paris.

LARSON N. L., Hill E. G., I960. Amine formation and metabolic activity of microorganisms in the ileum of young swine fed chlortetracycline. J. Bact., 80, 188-192.

Miciel M., Françors A. C., 1955. Relation entre l'influence des antibiotiques sur la croissance du Porc et l'inhibition des désaminases de la flore intestinale. C.R. Acad. Sci. Paris. 240, 808.

MOORE S., STEIN W. H., I954. A modified ninhydrin reagent for the photometric determination of amino acids and related compounds. J. Biol. Chem., 211, 907-91 r.

Salmon-Legagneur E., Micirel M., i955. Actions des antibiotiques sur porcelets allaités. Ann. Zootech, 4, $153-55^{-1}$

Salmon-Legagneur E., I96r. Les antibiotiques et la croissance du Porc. IX. Action de la spiramycine sur la croissance du porcelet avant et après le sevrage. Ann. Zootech., 10, 39-43.

SiebUrti J. M. N., I 960. Acrylic acid, and « antibiotic " principle in phacocystis blooms in antartic waters. Science, 132, 676-677.

Sieburth J. M. N., 196r. Antibiotic properties of acrylic acid, a factor in the gastrointestinal antibiosis of polar marine animals. J. Bact., 82, 72-79.

White-Stevens R. H., Pensack J. M., Stokstad E. L. R., Sieburth J. M. N., 1962. The effect of acrylic acid salts on growth of chicks. Poult. Sci., 41, 1909-1915.

Wallace H. D., Combs G. E., Ig62. Feed additives for growing-finishing swine. Mimeo S. 62-10 Florida Agric. Exp. Sta., I-4.

Wilber R. D., Catron D. V., QuinN L. Y., Speer C. V., Hays V. W., I960. Intestinal flora of the pig as influenced by diet and age. J. Nutr., 71, $168-75$. 